\title{
AGTR1 promoter hypermethylation in lung squamous cell carcinoma but not in lung adenocarcinoma
}

\author{
RUHUA CHEN $^{1 *}$, QINGXIAO HONG ${ }^{2 *}$, JIANZHONG JIANG $^{3 *}$, XIAOYING CHEN ${ }^{4 *}$, \\ ZHENHUAN JIANG $^{5^{*}}$, JINZHI WANG ${ }^{6 *}$, SHUNLIN LIU $^{7 *}$, SHIWEI DUAN ${ }^{4}$ and SHUNBIN SHI ${ }^{8}$
}

\begin{abstract}
${ }^{1}$ Department of Respiratory Medicine, Affiliated Yixing Hospital of Jiangsu University, Yixing, Jiangsu 214200;
${ }^{2}$ Laboratory of Behavioral Neuroscience, Ningbo Institute of Microcirculation and Henbane, Ningbo Addiction Research and Treatment Center, Ningbo, Zhejiang 315010; ${ }^{3}$ Department of Geriatrics, Affiliated Yixing Hospital of Jiangsu University, Yixing, Jiangsu 214200; ${ }^{4}$ Medical Genetics Center, School of Medicine, Ningbo University, Ningbo, Zhejiang 315211; ${ }^{5}$ Department of Orthopaedics, Affiliated Yixing Hospital of Jiangsu University, Yixing, Jiangsu 214200;

${ }^{6}$ Department of Cell Biology, School of Medicine, Soochow University, Suzhou, Jiangsu 215000; ${ }^{7}$ Department of Respiratory Medicine, The First Affiliated Hospital of Soochow University, Suzhou, Jiangsu 215006; ${ }^{8}$ Department of Thoracic Surgery, Affiliated Wujiang Hospital of Nantong University, Wujiang, Suzhou, Jiangsu 215000, P.R. China
\end{abstract}

Received December 12, 2016; Accepted August 3, 2017

DOI: $10.3892 / 01.2017 .6824$

\begin{abstract}
Aberrant DNA methylation is associated with non-small cell lung cancer (NSCLC), suggesting that gene promoter methylation may be a potential biomarker for the detection or risk prediction of NSCLC. The present study aimed to evaluate the potential usage of angiotensin II receptor type 1 (AGTR1) methylation in two major pathologic subtypes: Lung adenocarcinoma (LUAD) and lung squamous cell carcinoma (LUSC). Quantitative methylation-specific polymerase chain reaction was used to investigate the effect of AGTRI promoter methylation in the tumor and the paired adjacent non-tumor tissue samples from 42 patients with LUSC, and 69 with LUAD. The percentage of methylated reference was calculated and presented as the median (interquartile range 25th-75th percentile). The results of the current study revealed that there was significantly increased AGTRl promoter methylation in the tumor tissues compared with the paired adjacent non-tumor tissue [97.4 (57.22-130.5) vs. 85 (48.25-123); $\mathrm{P}=0.024]$. Furthermore, higher AGTR1 promoter methylation was observed in patients
\end{abstract}

Correspondence to: Dr Shunbin Shi, Department of Thoracic Surgery, Affiliated Wujiang Hospital of Nantong University, 3 Park Road, Wujiang, Suzhou, Jiangsu 215000, P.R. China

E-mail: 3319917810@qq.com

Dr Shiwei Duan, Medical Genetics Center, School of Medicine, Ningbo University, 818 Fenghua Road, Ningbo, Zhejiang 315211, P.R. China

E-mail: duanshiwei@nbu.edu.cn

*Contributed equally

Key words: angiotensin II receptor type 1, lung adenocarcinoma, lung squamous cell carcinoma, quantitative methylation specific polymerase chain reaction, promoter methylation with LUSC compared with LUAD (odds ratio $=2.483$; $95 \%$ confidence interval=1.125-5.480; $\mathrm{P}=0.023)$. Significant differences were identified in AGTR1 methylation between non-tumor and the tumor tissues in LUSC [113.5 (68.33-148.73) vs. 93.04 (45.94-140); $\mathrm{P}=0.008$ ]. In addition, the Cancer Genome Atlas data of 378 patients with LUSC and 477 with LUAD revealed an inverse correlation between gene expression and the methylation status of AGTRI promoter.. These data suggest that AGTRI hypermethylation is a promising biomarker to assist in LUSC detection and diagnosis.

\section{Introduction}

Lung cancer is a kind of malignancy that arises from epithelial cells. It is the leading cause of cancer-related death worldwide (1). There were nearly 1.8 million new patients and caused 159 million deaths in 2012, of which China accounted for more than one third (2). According to the size and appearance of the malignant cells, lung cancer is categorized as non small cell lung cancer (NSCLC) and small cell lung cancer (3). NSCLC represents approximately $85 \%$ of lung cancer (4), and it can be further subdivided into large cell carcinoma, lung adenocarcinoma (LUAD) and lung squamous cell carcinoma (LUSC). There are a lot of differences in molecular profiling, characteristics and therapeutic methods between LUAD and LUSC (5).

Promoter hypermethylation of the tumor suppressor genes has been recognized as an important factor in inducing oncogenesis (6). For example, SRY-box 17 (SOX17) methylation was found in $60.2 \%$ of primary lung cancer samples, and promoter methylation of SOX17 silenced gene expression, leading to the elimination of cell proliferation suppression in lung cancer (7). Identification of specific gene hypermethylation may explain the genomic instability and complexity of NSCLC and provide a basis for targeted therapy or risk prediction. 
AGTRI encodes the angiotensin II (Ang II) type I receptor that belongs to the family of G-protein coupled receptors (8). Ang II is a major effector controlling blood pressure in cardiovascular system and induces diverse signal transduction pathways such as the biphasic activation of Raf-1, MEK, and ERK via both Gq and Gi proteins (9). It regulates the aldosterone secretion and is involved in vascular remodeling, inflammation and endothelial dysfunction (10). Hypomethylation in the AGTR1 promoter had been validated to be inversely correlated with uric acid levels, which can be a significant risk predictor of essential hypertension (EH) (11). Besides, AGTR1 methylation had been extensively studied in human cancers, such as oral squamous cell carcinoma (12), colorectal cancer (13), breast cancer (14), ovarian cancer (15) and oral cancer (12). For example, several studies support a possible role for AGTR1 in regulating cell growth and proliferation during cancer development in breast cancer (16), which was shown to be amplified and overexpressed in 10-20\% of breast cancer cases, and was even markedly overexpressed more than 100 fold (14). Besides, the AGTR 1 promoter methylation is associated with oral squamous cell carcinoma (OSCC) development (12). And AGTR1 was also validated in stool DNA with a high detection sensitivity for noninvasive diagnosis of colorectal cancer (13). In addition, DNA methylation microarray datasets showed that methylation of AGTRl might be an effective biomarker for NSCLC diagnosis (17).

Since the detection of AGTR1 methylation in different pathologic subtypes and stages have not been conducted, we used LUSC and LUAD samples to study the effects of AGTRI methylation on the risk of the disease in this study.

\section{Materials and methods}

Patients. Tumor tissues and paired adjacent non-tumor tissues from 111 patients were collected from Affiliated Wujiang Hospital of Nantong University (Jiangsu, China) between August 2010 and October 2013. There were 73 male and 38 female patients with a mean age of $63.59 \pm 10.19$ years (range, 33-82 years), including 42 patients with LUSC and 69 patients with LUAD. Clinical pathological data and isoforms were obtained from the patients' medical records and pathology files. Clinical stage was classified by the third edition of the American College of Chest Physicians (ACCP) Lung Cancer Guidelines (LC III) (18). The study protocol was approved by the Ethics Committee of Affiliated Wujiang Hospital of Nantong University. All the patients had signed the written informed consent forms.

DNA extraction and bisulphite conversion. DNA was isolated from the formalin-fixed and paraffin-embedded (FFPE) cancer sample using QIAamp DNA FFPE Tissue Kit (Qiagen Inc., Hilden, Germany). DNA concentrations were measured using the NanoDrop 1000 spectrophotometer (Thermo Fisher Scientific Inc., Waltham, MA, USA). Then DNA were converted using the EZ DNA Methylation-Gold Kit ${ }^{\mathrm{TM}}$ (Zymo Research Corporation, Irvine, CA, USA).

Quantitative methylation-specific PCR. SYBR green-based quantitative methylation-specific PCR (qMSP) was conducted to detect the methylation level. PCR was carried out in a final volume of $20 \mu \mathrm{l}$ containing $5 \mu \mathrm{l} \mathrm{SYBR} \operatorname{mix}, 4 \mu \mathrm{l} \mathrm{H}_{2} \mathrm{O}, 0.5 \mu \mathrm{l}$ primer and $0.5 \mu 1$ modified DNA. PCR amplification were run in triplicate for every sample, and the reactions was performed on Light Cycler 480 system (Roche Applied Science, Mannheim, Germany) under the following conditions: $10 \mathrm{~min}$ of denaturation at $95^{\circ} \mathrm{C}$ followed by 45 cycles of $20 \mathrm{sec}$ at $95^{\circ} \mathrm{C}$, $20 \mathrm{sec}$ at $58^{\circ} \mathrm{C}$ and $30 \mathrm{sec}$ at $72^{\circ} \mathrm{C}$. Melting curves system was as follows: $15 \mathrm{sec}$ at $95^{\circ} \mathrm{C}, 1 \mathrm{~min}$ at $58^{\circ} \mathrm{C}, 95^{\circ} \mathrm{C}$ continuous prior to drop the temperature to $40^{\circ} \mathrm{C}$ for $4 \mathrm{~min}$. The Actin Beta gene $(A C T B)$ was used as an internal reference by amplifying non-CpG sequences. Results with cycle threshold values (Ct values) of $A C T B>40$ were defined as detection failures. M.SssI catalyzes the deamination of target cytosine to uracil to generate DNAs that differ only in their $\mathrm{CpG}$ methylation status (19). The methylated DNA was prepared to be the positive internal control. Each set of amplifications included a positive control, a negative control, and a non-template control.

The sequences of primers for target and internal reference genes were summarized in Table I, and the genomic location of AGTRl gene was shown in Fig. 1A. Some of the PCR products were analysed using to the Qsep100 DNA Analyzer (Bioptic Inc, Taiwan, China) to validate the methylation status, and ultimately the visible peaks were exported by the Q-analyzer software (Fig. 1B). Besides, some of the products were sequenced randomly using the Applied Bio systems ${ }^{\circledR}$ 3730 DNA Analyzer (Applied Biosystems, Warrington, UK) to confirm a complete bisulphite conversion (Fig. 1B).

Statistical analysis. For each sample the comparative $\mathrm{Ct}(\Delta \Delta \mathrm{Ct})$ method was used to determine the relative methylation values. And the percentage of methylated reference (PMR) was shown by using the following formula (substituted the actual methylation value here): [(gene/AGTRl) sample/(gene/AGTRl) positive] x $100 \%$. The median of PMR (97.4) was set as the cutoff value and defined methylation as hypermethylation (positive) and hypomethylation (negative) subgroups. Statistical analyses were performed using SPSS 18.0 version (SPSS Inc., Chicago, IL, USA). The wilcoxon signed ranks test was used to determine the difference of the methylation index between tumor tissues and non-tumor tissues. $\chi^{2}$ test was used to evaluate the association between promoter methylation and clinical parameters. Overall survival in relation to methylation status was calculated by Kaplan-Meier survival curves, and survival differences were assessed in the log-rank test. $\mathrm{P}<0.05$ was considered to indicate statistical significance.

\section{Results}

The result of sequenced PCR products showed that all the non-CpG cytosines were converted to thymine, whereas the cytosines of CG dinucleotide remained unchanged (Fig. 1). And the results of capillary electrophoresis experiments showed the length of products were correct.

As the results revealed, there existed statistical differences in AGTRI methylation between tumor tissues and the adjacent non-tumor tissues (PMR: 97.4 vs. 85, $\mathrm{P}=0.024$ ). A subgroup analysis indicated that there was a significantly induced hypermethylation of AGTRl in LUSC tumors $(\mathrm{P}=0.008)$ but not in LUAD tumors ( $\mathrm{P}=0.449$, Fig. 2). 
Table I. Primer sequences for quantitative methylation-specific polymerase chain reaction.

\begin{tabular}{lllcc}
\hline Gene & \multicolumn{1}{c}{ Forward primer $\left(5^{\prime}-3^{\prime}\right)$} & \multicolumn{1}{c}{ Reverse primer $\left(5^{\prime}-3^{\prime}\right)$} & Product $(\mathrm{bp})$ & $\mathrm{Tm}\left({ }^{\circ} \mathrm{C}\right)$ \\
\hline AGTR1 & GGAGGAGGAGGGAATGTAA & CCTATCACTCGCTACTACCT & 142 & 58 \\
ACTB & TGGTGATGGAGGAGGTTTAGTAAGT & AACCAATAAAACCTACTCCTCCCTTAA & 133 & 58 \\
\hline
\end{tabular}

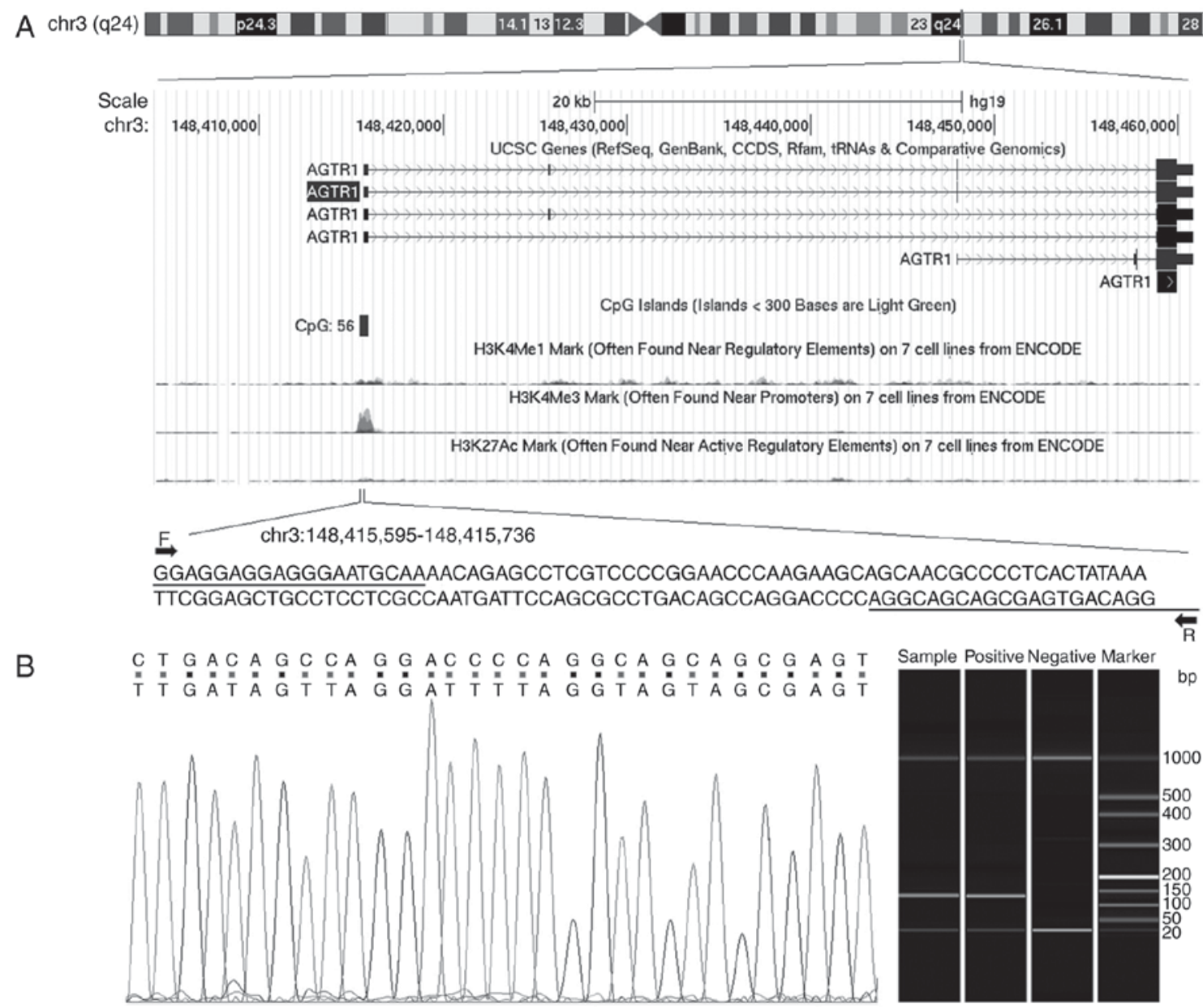

Figure 1. AGTR1 and the validation for sequencing and electrophoretic results. (A) The location of AGTR1 on the chromosome and the sequences of primer for target gene. (B) The top row of sequencing validation result represents the original gene sequence, and the second row shows the converted one. The electrophoresis experiments reveal the results of methylated sample, the positive internal control and the negative control.

Further correlation analysis of clinicopathologic characteristics in patients with promoter methylation status was also performed (Table II). There was higher AGTRI promoter methylation in LUSC patients than LUAD (Odds ratio $=2.483,95 \% \mathrm{CI}=1.125-5.480, \mathrm{P}=0.023$ ). In addition, AGTR1 promoter methylation was not associated with gender, age, smoking history, clinical stage and lesion location in NSCLC (Table II).

Previous study showed that AGTRl is predictor of progression-free survival (PFS) and response to advanced/metastatic breast cancer (20). However, the correlation of AGTRI methylation with overall survival of cancer was unknown. In this study, we examined the overall survival of individuals according to AGTRl PMR value, and no significant difference was observed between LUSC and the LUAD patients ( $\mathrm{P}=0.293$, Fig. 3).
To further reveal the correlation between expression and methylation status of AGTRI in LUSC and LUAD, the MEXPRESS system (21) (http://mexpress.be) was used. And the Cancer Genome Atlas (TCGA) data of 378 LUSC and 477 LUAD patients (21) indicated a lower expression in cancer tissues, supporting an inverse correlation between gene expression and AGTRl methylation.

\section{Discussion}

NSCLC is a huge threat to human health, and the study of the methylation and its relation to NSCLC is a field of growing interest. AGTR1 acts as a novel component of the renin-angiotensin system (RAS) to affect the blood pressure and heart hypertrophy (15), targeting of renin-angiotensin system (RAS) through AGTR1 blocker is associated with the resistance 
Table II. Association between gene methylation and clinicopathological characteristics in patients with lung cancer.

\begin{tabular}{|c|c|c|c|c|c|}
\hline Variables & $\mathrm{n}$ & $\begin{array}{c}A G T R 1 \\
\text { hypermethylation }\end{array}$ & $\begin{array}{c}A G T R l \\
\text { hypomethylation }\end{array}$ & OR $(95 \% \mathrm{CI})$ & P-value \\
\hline \multicolumn{6}{|l|}{ Gender } \\
\hline Male & 73 & 38 & 35 & $1.206(0.550-2.645)$ & 0.639 \\
\hline Female & 38 & 18 & 20 & 1 & \\
\hline \multicolumn{6}{|l|}{ Age (years) } \\
\hline$\leq 65$ & 62 & 31 & 31 & $0.960(0.454-2.031)$ & 0.915 \\
\hline$>65$ & 49 & 25 & 24 & 1 & \\
\hline \multicolumn{6}{|c|}{ Smoking history } \\
\hline Nonsmoker & 50 & 26 & 24 & $1.119(0.530-2.366)$ & 0.768 \\
\hline Smoker & 61 & 30 & 31 & 1 & \\
\hline \multicolumn{6}{|c|}{ Histological type } \\
\hline LUSC & 42 & 27 & 15 & $2.483(1.125-5.480)$ & 0.023 \\
\hline LUAD & 69 & 29 & 40 & 1 & \\
\hline \multicolumn{6}{|l|}{ Clinical stage } \\
\hline $\mathrm{I}+\mathrm{II}$ & 88 & 41 & 47 & $0.465(0.179-1.209)$ & 0.112 \\
\hline III+IV & 23 & 15 & 8 & 1 & \\
\hline \multicolumn{6}{|c|}{ Tumor location } \\
\hline Left lung & 46 & 25 & 21 & $1.306(0.612-2.784)$ & 0.490 \\
\hline Right lung & 65 & 31 & 34 & & \\
\hline
\end{tabular}

LUSC, lung squamous carcinoma; LUAD, lung adenocarcinoma; The median of percentage of methylated reference (PMR) (97.4\%) was set as the cutoff value, the PMR >97.4\% was considered to be hypermethylation and the PMR $\leq 97.4 \%$ was considered to be hypomethylation. Bold fonts displayed the statistical differences.

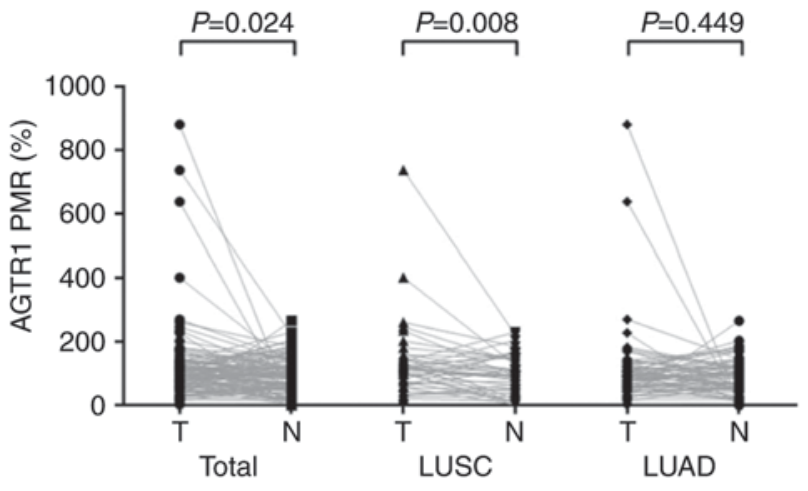

Figure 2. Comparisons of methylation levels of AGTR1 between tumor tissues and paired adjacent non-tumor tissue.

phenomenon in treatment of resistant breast cancers. And now the AGTRl blocker has been applied in the clinical oncology, such as the losartan for breast cancer (22) and candesartan for prostate cancer (23). And now emerging evidences showed that AGTR1 regulates the cell proliferation during cancer development, and promotes tumor invasion, migration, metastasis and angiogenesis (24). In consideration of the unfavorable treatment status of NSCLC, detection of biomarkers like the AGTRl is really necessary and urgent.

In the present study, DNA methylation differences of AGTRl between 111 paired tumor sample and adjacent non-tumor tissues were assessed using quantitative

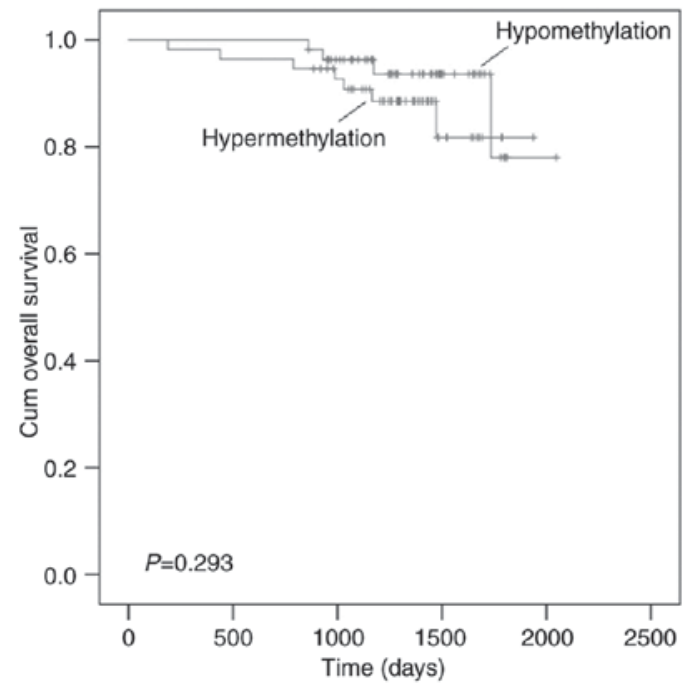

Figure 3. Kaplan-Meier curve for cum overall survival of individuals.

methylation-specific PCR. We identified significant differences in AGTRl methylation between tumor tissues and the adjacent non-tumor tissues, this observation lead us to suggest that $\mathrm{CpG}$ island methylation phenotype in AGTRl may be an early event during NSCLC development. Besides, AGTRI were hypermethylated in LUSC but not in LUAD, and there were significant differences in AGTR1 promoter methylation 
between LUAD and LUSC patients, suggesting a potential usage of this epigenetic biomarker in the diagnosis of LUSC. In addition, we found that AGTRl promoter methylation was not associated with gender, age, smoking history, clinical stage and lesion location in NSCLC. And research on the data of TCGA suggested a negative correlation between gene expression and AGTRl methylation.

Our finding of AGTR1 promoter methylation as a diagnostic marker of NSCLC was consistent with a study of high-throughput DNA methylation microarray dataset for Chinese Han NSCLC retrospective cohort which showed the significant association between methylation and NSCLC (17). This was also consistent with the result of TCGA for LUSC and LUAD patients, suggesting that AGTRl might be associated with increased risk of NSCLC.

Our results showed that there were significant differences in AGTR1 promoter methylation between LUAD and LUSC patients, and there was a significantly induced hypermethylation of AGTR1 in LUSC tumors compared with the normal tissues but not in LUAD. Previous study declared differentially methylated genes were specially differential methylation in unique cancer, and the DNA methylation correlation network had been built based on the methylation correlation, such as seven biomarkers (PCDHB15, IGF1, PRRT1, CYGB, WBSCR17, ACTG2 and GYPC) can distinguish the breast cancer into high-risk group and low-risk group. Likewise, different methylation status in eight biomarkers (ZBTB32, GPSM1, SALL1, OR51B4, MAGEA8, SALL3, CCL8 and $T M E F F 2$ ) in colon cancer showed the different risk level (25). So the methylated AGTR1 might be identified as the specific driver gene for LUSC, which might be implicated in cancer type specific pathway and be expected to be used to explain part of the heterogeneity between these two NSCLC types.

NSCLC was regarded as a complex diseases, and some clinicopathologic characteristics were involved in cancer, such as the smoking history, which was identified as the main risk factor of lung cancer (26), and association between tobacco smoking and promoter DNA hypermethylation had been demonstrated for several genes, such as the transcription factor 21 (TCF21) and MicroRNA Let-7a-3 (27). The previous study on primary lung cancer cases in China $(\mathrm{N}=40,022)$ showed that males were 1.5 times more likely to have lung cancer than females (28), but the incidence of lung cancer among women was rising exponentially as a consequence of recent changes in gender-specific smoking patterns. Besides, women with lung cancer were diagnosed at younger ages than men, suggesting the different gender and age susceptibility existed in patients (29). Notablely, the clinical stages were important prognostic factors in NSCLC. The advanced disease stages affected survival negatively (30). Besides, even though apparently symmetric, previous study showed that higher incidence of lung cancer was found on the right side (31), just as the breast cancer, which was about 5\% more likely to be diagnosed in the left breast than the right (32). Breast cancer that arising on different sides of the body presented different cancer traits inferred from methylation and expression profiles (31), and this result contributed to serve as proof of principle for other bilateral cancers like the lung cancer. In our study, AGTRl promoter methylation was shown likely to be not linked with gender, age, smoking history, clinical stage and tumor location. But the further verification is required to ensure the results considering of the limitation factors existed in our experimental design.

In the present study, we enrolled the relatively large cohorts that contained 111 patients, and we collected the precious surgery tissues to perform the survey by general methodology that easily to be applied to different cancer subtypes. However, there existed several limitations here. Firstly, because of the limited time, the large cell carcinoma was not included in our study, the study design may still be too simple to uncover the complicated trait of NSCLC. Secondly, because of the limited amount of sample, we did not detect the expression of different methylated AGTR1 and replaced by a database analysis of TCGA, which showed the negative relevance between methylation and expression of AGTR1. However, one previous studies showed there was no correlation between the degree of methylation and mRNA abundance of AGTR1 in early gestation amnion and placenta (33), so the further studies are needed. Thirdly, the mechanisms by which AGTRl methylation affect the LUSC remained largely elusive. Fourthly, we just detect the part of the content area, it could be possible that some more $\mathrm{CpG}$ sites are required for gene function, besides, additional epigenetic alteration, such as the histone modifications that closely connected with the gene methylation are needed for study on NSCLC progression. In addition, only the AGTRI gene was investigated in the present study, and additional relevant genes should be explored in the future.

In conclusion, our findings of the association between AGTR 1 methylation and LUSC provided a potential biomarker for detection, diagnosis and risk prediction for LUSC.

\section{Acknowledgements}

The research and publication was supported by the grants from the National Natural Science Foundation of China (31100919, 81371469), K. C. Wong Magna Fund of the Ningbo University, National Natural Science Foundation for the Youth of China (81402220) and Suzhou Planning Project of Science and Technology (SYS201301).

\section{References}

1. Barlési F, Giaccone G, Gallegos-Ruiz MI, Loundou A, Span SW, Lefesvre P, Kruyt FA and Rodriguez JA: Global histone modifications predict prognosis of resected non small-cell lung cancer. J Clin Oncol 25: 4358-4364, 2007.

2. McGuire S: World cancer report 2014. Geneva, Switzerland: World health organization, international agency for research on cancer, WHO press, 2015. Adv Nutr 7: 418-419, 2016.

3. Collins LG, Haines C, Perkel R and Enck RE: Lung cancer: Diagnosis and management. Am Fam Physician 75: 56-63, 2007.

4. NSCLC Meta-analysis Collaborative Group: Preoperative chemotherapy for non-small-cell lung cancer: A systematic review and meta-analysis of individual participant data. Lancet 383: 1561-1571, 2014.

5. Zhan C, Yan L, Wang L, Sun Y, Wang X, Lin Z, Zhang Y, Shi Y, Jiang W and Wang Q: Identification of immunohistochemical markers for distinguishing lung adenocarcinoma from squamous cell carcinoma. J Thorac Dis 7: 1398-1405, 2015.

6. Hong Q, Li Y, Chen X, Ye H, Tang L, Zhou A, Hu Y, Gao Y, Chen R, Xia Y and Duan S: CDKN2B, SLC19A3 and DLEC1 promoter methylation alterations in the bone marrow of patients with acute myeloid leukemia during chemotherapy. Exp Ther Med 11: 1901-1907, 2016. 
7. Yin D, Jia Y, Yu Y, Brock MV, Herman JG, Han C, Su X, Liu Y and Guo M: SOX17 methylation inhibits its antagonism of Wnt signaling pathway in lung cancer. Discov Med 14: 33-40, 2012

8. Haas U, Sczakiel G and Laufer SD: MicroRNA-mediated regulation of gene expression is affected by disease-associated SNPs within the 3'-UTR via altered RNA structure. RNA Biol 9: 924-937, 2012

9. Tsygankova OM, Peng M, Maloney JA, Hopkins $\mathrm{N}$ and Williamson JR: Angiotensin II induces diverse signal transduction pathways via both $\mathrm{Gq}$ and $\mathrm{Gi}$ proteins in liver epithelial cells. J Cell Biochem 69: 63-71, 1998.

10. BrietM,BarhoumiT,MianMOR,CoelhoSC,OuerdS,Rautureau Y, Coffman TM, Paradis P and Schiffrin EL: Aldosterone-induced vascular remodeling and endothelial dysfunction require functional angiotensin type 1a receptors. Hypertension 67: 897-905, 2016

11. Fan R, Mao S, Zhong F, Gong M, Yin F, Hao L and Zhang L: Association of AGTR1 promoter methylation levels with essential hypertension risk: A matched case-control study. Cytogenet Genome Res 147: 95-1029, 2015.

12. Foy JP, Pickering CR, Papadimitrakopoulou VA, Jelinek J, Lin SH, William WN Jr, Frederick MJ, Wang J, Lang W, Feng L, et al: New DNA methylation markers and global DNA hypomethylation are associated with oral cancer development. Cancer Prev Res (Phila) 8: 1027-1035, 2015.

13. Carmona FJ, Azuara D, Berenguer-Llergo A, Fernández AF, Biondo S, de Oca J, Rodriguez-Moranta F, Salazar R, Villanueva A, Fraga MF, et al: DNA methylation biomarkers for noninvasive diagnosis of colorectal cancer. Cancer Prev Res (Phila) 6: 656-665, 2013.

14. Rhodes DR, Ateeq B, Cao Q, Tomlins SA, Mehra R, Laxman B, Kalyana-Sundaram S, Lonigro RJ, Helgeson BE, Bhojani MS, et al: AGTR1 overexpression defines a subset of breast cancer and confers sensitivity to losartan, an AGTR1 antagonist. Proc Natl Acad Sci USA 106: 10284-10289, 2009.

15. Bi FF, Li D, Cao C, Li CY and Yang Q: Regulation of angiotensin II type 1 receptor expression in ovarian cancer: A potential role for BRCA1. J Ovarian Res 6: 89, 2013.

16. Du N, Feng J, Hu LJ, Sun X, Sun HB, Zhao Y, Yang YP and Ren H: Angiotensin II receptor type 1 blockers suppress the cell proliferation effects of angiotensin II in breast cancer cells by inhibiting AT1R signaling. Oncol Rep 27: 1893-1903, 2012

17. Guo S, Yan F, Xu J, Bao Y, Zhu J, Wang X, Wu J, Li Y, $\mathrm{Pu} \mathrm{W}$, Liu Y, et al: Identification and validation of the methylation biomarkers of non-small cell lung cancer (NSCLC). Clin Epigenetics 7: 3, 2015

18. Lewis SZ, Diekemper R and Addrizzo-Harris DJ: Methodology for development of guidelines for lung cancer: Diagnosis and management of lung cancer, 3rd ed: American college of chest Physicians evidence-based clinical practice guidelines. Chest 143 (5 Suppl): 41S-50S, 2013.

19. Hughes S and Jones JL: The use of multiple displacement amplified DNA as a control for methylation specific PCR, pyrosequencing, bisulfite sequencing and methylation-sensitive restriction enzyme PCR. BMC Mol Biol 8: 91, 2007.
20. Salvador J, Manso L, de la Haba J, Jaen A, Ciruelos E, de Villena MC, Gil M, Murias A, Galan A, Jara C, et al: Final results of a phase II study of paclitaxel, bevacizumab, and gemcitabine as first-line therapy for patients with HER2-negative metastatic breast cancer. Clin Transl Oncol 17: 160-166, 2015.

21. Koch A, De Meyer T, Jeschke J and Van Criekinge W: MEXPRESS: Visualizing expression, DNA methylation and clinical TCGA data. BMC Genomics 16: 636, 2015.

22. Namazi S, Sahebi E, Rostami-Yalmeh J, Jaberipour M, Razmkhah M, Hosseini A and Arabsolghar R: Effect of angiotensin receptor blockade on prevention and reversion of tamoxifen-resistant phenotype in MCF-7 cells. Tumour Biol 36: 893-900, 2015.

23. Alhusban A, Al-Azayzih A, Goc A, Gao F, Fagan SC and Somanath PR: Clinically relevant doses of candesartan inhibit growth of prostate tumor xenografts in vivo through modulation of tumor angiogenesis. J Pharmacol Exp Ther 350: 635-645, 2014

24. Chen X, Meng Q, Zhao Y, Liu M, Li D, Yang Y, Sun L, Sui G, Cai L and Dong X: Angiotensin II type 1 receptor antagonists inhibit cell proliferation and angiogenesis in breast cancer. Cancer Lett 328: 318-324, 2013.

25. Zhang C, Zhao H, Li J, Liu H, Wang F, Wei Y, Su J, Zhang D, Liu T and Zhang Y: The identification of specific methylation patterns across different cancers. PLoS One 10: e0120361, 2015.

26. Word B, Lyn-Cook LE Jr, Mwamba B, Wang H, Lyn-Cook B and Hammons G: Cigarette smoke condensate induces differential expression and promoter methylation profiles of critical genes involved in lung cancer in NL-20 lung cells in vitro: Short-term and chronic exposure. Int J Toxicol 32: 23-31, 2013.

27. Lyn-Cook L, Word B, George N, Lyn-Cook B and Hammons G: Effect of cigarette smoke condensate on gene promoter methylation in human lung cells. Tob Induc Dis 12: 15, 2014.

28. Chen K, Wang PP, Sun B, Li Q, Perruccio A, Power D, Wang C, He M, M H, Shibei Y, et al: Twenty-year secular changes in sex specific lung cancer incidence rates in an urban Chinese population. Lung Cancer 51: 13-19, 2006.

29. Isla D, Majem M, Viñolas N, Artal A, Blasco A, Felip E, Garrido P, Remón J, Baquedano M, Borrás JM, et al: A consensus statement on the gender perspective in lung cancer. Clin Transl Oncol 19: 527-535, 2017

30. Urvay SE, Yucel B, Erdis E and Turan N: Prognostic factors in stage III non-small-cell lung cancer patients. Asian Pac J Cancer Prev 17: 4693-4697, 2016.

31. Campoy EM, Laurito SR, Branham MT, Urrutia G, Mathison A Gago F, Orozco J, Urrutia R, Mayorga LS and Roqué M: Asymmetric cancer hallmarks in breast tumors on different sides of the body. PLoS One 11: e0157416, 2016.

32. Perkins CI, Hotes J, Kohler BA and Howe HL: Association between breast cancer laterality and tumor location, United States, 1994-1998. Cancer Causes Control 15: 637-645, 2004.

33. Sykes SD, Mitchell C, Pringle KG, Wang Y, Zakar T and Lumbers ER: Methylation of promoter regions of genes of the human intrauterine Renin Angiotensin system and their expression. Int J Endocrinol 2015: 459818, 2015. 